\title{
CIENCIA EN EL BARROCO ESPAÑOL: NUEVAS FUENTES DOCUMENTALES DE JERÓNIMO DE AYANZ
}

\author{
Nicolás García Tapia \\ Real Academia de Bellas Artes de la Purísima Concepción, Valladolid \\ Carlos Jiménez Muñoz \\ IES Valle del Alberche, Navaluenga (Ávila) \\ cjimenezmuno@educa.jcyl.es \\ Andrés Martínez de Azagra Paredes \\ E.T.S. de Ingenierías Agrarias de Palencia, Universidad de Valladolid \\ amap@iaf.uva.es
}

Recibido: 9 septiembre 2014; Aceptado: 8 mayo 2015.

\begin{abstract}
Cómo citar este artículo/Citation: García Tapia, Nicolás; Carlos Jiménez Muñoz y Andrés Martínez de Azagra Paredes (2016), "Ciencia en el Barroco español: nuevas fuentes documentales de Jerónimo de Ayanz", Asclepio 68 (1): p122. doi: http://dx.doi.org/10.3989/ asclepio.2016.01

RESUMEN: En la vida de Jerónimo de Ayanz hay un lapso de tiempo, desde noviembre de 1611 hasta su muerte, en marzo de 1613, del que no disponíamos de ninguna fuente documental. Recientemente, un impreso adquirido por la Biblioteca Nacional de España, y que hemos fechado en 1612, revela que el técnico estuvo activo hasta sus últimos días y nos muestra una nueva faceta suya: la experimentación científica. Este documento incluye una carta impresa dirigida al príncipe Emanuel Filiberto de Saboya. En la misiva, Ayanz presenta al príncipe un capítulo de un libro científico desconocido que estaba escribiendo en el que, a través de la experimentación, trata asuntos como la compulsión de los elementos, la existencia del vacío, el movimiento perpetuo, la esfera ígnea o la caída de los cuerpos. Nuestro objetivo es presentar este valioso documento y exponer los experimentos e innovadoras teorías científicas que Ayanz plantea.
\end{abstract}

PALABRAS CLAVE: Jerónimo de Ayanz; Emanuel Filiberto de Saboya; Movimiento perpetuo; Esfera de fuego; Caída de los graves.

\section{SCIENCE IN THE SPANISH BAROQUE: NEW DOCUMENTARY SOURCES OF JERÓNIMO DE AYANZ}

ABSTRACT: Recently, a printed document of Jerónimo de Ayanz, acquired by the Biblioteca Nacional de España (the National Library of Spain), has revealed that this inventor was active until the very last days of his life and shows his interest in technology, research and science. The document, addressed to the Prince Emmanuel Philibert of Savoy, is one chapter of a book, of which no copies are known, that Ayanz had prepared in 1612 and that he probably wanted to publish with the support of the Prince. In this paper we present and comment this important text, where the author discusses central scientific subjects of his time such as the compulsory movement of the elements, the existence of vacuum, the possibility of perpetual motion, the sphere of fire and falling bodies.

KEY WORDS: Jerónimo de Ayanz; Emmanuel Philibert of Savoy; Perpetual motion; Sphere of fire; Falling bodies.

Copyright: () 2016 CSIC. Este es un artículo de acceso abierto distribuido bajo los términos de la licencia Creative Commons Attribution (CC BY) España 3.0. 


\section{INTRODUCCIÓN}

En octubre de 2013, la Biblioteca Nacional ha adquirido un impreso desconocido del inventor navarro Jerónimo de Ayanz y Beaumont (1553-1613) procedente de un particular ${ }^{1}$. Este texto de principios del siglo XVII va dirigido a Emanuel Filiberto de Saboya (1568-1624) e incluye un capítulo completo de un libro científico desconocido que Ayanz estaba redactando. El documento consta de 8 hojas impresas en formato folio, tamaño $28,5 \times 20 \mathrm{~cm}$, con siete dibujos del autor y encuadernadas en pergamino moderno (figuras 1-9).

Ayanz, el autor del impreso, encarna al hombre universal del Renacimiento, pues destaca en la música, en la pintura, en la milicia y en otras disciplinas. Sin embargo, sus mejores trabajos los realiza dentro del campo de la técnica. El privilegio de invención de Ayanz ${ }^{2}$, otorgado por el monarca Felipe III en 1606, es el más importante de todos los conocidos (García Tapia, 2001, pp. 181). Este documento incluye algunos de los proyectos que Ayanz ejecuta mientras desempeña el cargo de Administrador General de Minas de España (García Tapia, 2001, pp. 124), siendo los más destacados aquéllos que funcionan con vapor de agua. Así, proyecta un eyector para extraer el aire contaminado de las minas, la primera máquina de aire acondicionado conocida y un sistema para desaguar las minas con un funcionamiento similar al que desarrollará Thomas Savery casi un siglo después (García Tapia, 2001, pp. 213-225). El documento de patente incluye también inventos tan variados como una balanza capaz de pesar "la pierna de una mosca», hornos de fundición, instrumentos para destilar el agua del mar, un sistema para medir el rendimiento de las máquinas, presas de arco y de bóveda, bombas de husillo y de achique para barcos y molinos de sangre, de rodillos y de viento (García Tapia, 2001, pp. 182-205). Además, en el mismo privilegio de invención, Ayanz perfecciona las campanas de bucear existentes, diseña el primer precedente de un submarino e idea múltiples sistemas para que un hombre pueda estar sumergido en el agua sin poner en peligro su vida (García Tapia, 2001, pp. 159-178). En total, Ayanz es autor de más de cincuenta invenciones, patentadas en 1606 adelantándose a las que serían corrientes en la época de la Revolución Industrial dos siglos después (García Tapia, 1992, pp. 184).

La contribución de Ayanz al campo de la técnica es asombrosa, quedando sus teorías científicas en un segundo plano. Sin embargo, el impreso descubierto da una nueva dimensión a esta faceta y enriquece el elenco de documentos científicos de Ayanz. Entre dichos documentos encontramos el Informe sobre las agujas de marear ${ }^{3}$, en el que el técnico navarro demuestra poseer una avanzada explicación teórica del magnetismo y el único impreso conocido hasta la reciente aparición de la carta, la Respuesta de D. Gerónimo de Ayanz, Comendador de Ballesteros, de la Orden de Calatrava a lo que el Reyno le preguntó acerca de las minas de estos Reynos, y del metal Negrillo de Potosí ${ }^{4}$. A éstos podemos añadir el Discurso para uso de los baxeles (García Tapia, 2001, pp. 242), que aún no ha sido localizado, pero del que conocemos parte de su contenido por referencias en el Informe sobre las agujas de marear.

La importancia del documento dirigido a Emanuel Filiberto de Saboya se debe a que en él están tratados muchos de los asuntos que inquietan a los científicos de su época, como la compulsión de los elementos, la esfera ígnea, las tesis del horror vacui o el movimiento perpetuo. Además, hace referencia a algunos de los temas tratados en otros capítulos, lo que sugiere que el libro del que formaría parte sería un documento científico de la máxima relevancia.

\section{CIRCUNSTANCIAS DEL CAPÍTULO}

El impreso de Ayanz que presentamos, dedicado al príncipe Emanuel Filiberto de Saboya cuando ya era "Generalísimo del Mar», constituye un capítulo de un libro desconocido del técnico navarro.

\section{DATACIÓN}

La toma de posesión de Emanuel Filiberto de Saboya como Capitán General del Mar tuvo lugar el 4 de diciembre de 1612 (Fernández Duro, 1885, pp. 209), pero el nombramiento fue en enero del mismo año ${ }^{5}$. La dedicatoria está, pues, hecha a partir de esta fecha y antes de la muerte de Ayanz, el 23 de marzo de 1613 (García Tapia, 2001, pp. 264). Así, el texto fue realizado en los últimos años de su vida, que fue el periodo dedicado a discusiones científicas, como el de la aguja magnética presentado en noviembre de 1611, dos meses después de haber dejado de explotar las minas de su compañía. Entonces tenía su domicilio en Madrid (García Tapia, 2001, pp. 237-243).

Este impreso está fechado en una etapa de la vida de Ayanz de la que no disponíamos de ningún documento y nos muestra que estuvo activo hasta los últimos días de su vida, confirmándose la suposición de García Tapia (2001, pp. 264): 
Desde septiembre de 1611, en que aparece el último documento que hemos encontrado relacionado con la explotación de las minas, hasta marzo de 1613 en que Ayanz hizo su testamento, no existen indicios documentales sobre iniciativas suyas de ningún tipo, lo que es raro en una persona tan inquieta como el comendador.

\section{CONTEXTO}

Es el resultado de una discusión científica, propia de la época, ante personajes influyentes (caso del príncipe Emanuel Filiberto de Saboya) y eruditos (como el «Doctor Fioqueto $\left.{ }^{6} »\right)$. Esto indica que Ayanz estaba en contacto con los medios científicos de la época pero, como veremos, su discusión no era superficial, sino comprobada con instrumentos científicos que el autor había ideado y construido.

\section{ANOTACIONES AL MARGEN}

Apenas conocemos las vicisitudes posteriores del impreso, pero en los márgenes del mismo aparecen anotaciones que nos aportan algunos indicios.

En la primera hoja del impreso (figura 1), en el margen izquierdo hay un signo de la Compañía de Jesús invertido, posiblemente al aplicar un secante. Los Jesuitas eran los gestores de la enseñanza en muchas disciplinas ( $\mathrm{Na}$ varro, 1996, pp. 16), por lo que no es de extrañar que se interesasen por un documento como el del técnico navarro. En el margen derecho, está marcado un sello casi imperceptible e, inmediatamente debajo, encontramos una glosa poco legible y aparentemente dedicada al autor: "Pero métete allá dentro [...] Pícaro no [...] me salgas». El lector, posiblemente un jesuita, discrepaba, al parecer indignado, de unas ideas que, como veremos, desmontaban algunos principios de origen aristotélico fuertemente arraigados en determinados medios, sobre todo eclesiásticos. En el margen superior, aparecen sílabas y letras sueltas que transcriben algunos fragmentos del texto, lo que invita a pensar que el documento pudo haber sido utilizado para instruir en la escritura.

En los siguientes folios, algunos de ellos deteriorados por el tiempo, no aparece ninguna anotación, hasta que en la cuarta hoja (figura 4) volvemos a encontrar letras manuscritas y, de nuevo, la palabra «Pícaro». En el margen izquierdo de la séptima hoja (figura 7) aparece una ingenua ilustración de un hombre con la indumentaria militar del siglo XVII y en la última hoja (figura 9), el lector, posiblemente escandalizado, termina con una oración, como si no quisiera "caer» en la «tentación» del «mal» que había leído: «Señor nuestro ruega por nosotros, no nos dejes caer en la tentación, líbranos del mal. Amén».

\section{OTROS PROPIETARIOS POSIBLES}

El impreso pudo haber pertenecido a la antigua Biblioteca Mugartegui en Marquina procedente posiblemente de la Biblioteca-Archivo de D. Javier María de Munibe, conde de Peñaflorida, fundador de la Real Sociedad Bascongada de Amigos del País. En la hoja de guarda anterior aparece grabado un ex libris de José Mañas Martínez.

\section{ANÁLISIS DEL CONTENIDO}

\section{"Como no se puede hazer movimiento perpetuo"}

La búsqueda del móvil perpetuo era un afán recurrente en los círculos científicos de la época. Al respecto y en España, son especialmente ingeniosos dos artificios proyectados en la segunda mitad del siglo XVI por un técnico de Medina del Campo, Francisco Lobato, que los denomina "Máquina gravísima y delicada» (García-Diego y García Tapia, 1990, pp. 47-48) y "Canal de agua que vuelve» (García-Diego y García Tapia, 1990, pp. 87-90).

Ayanz, por su parte, niega que sea posible alcanzar este objetivo y atribuye el intento de hacer movimiento perpetuo a la «soberbia de los hombres» y a su «ignorancia», situándolo en la línea de querer hacer oro y plata, asegurando que es engañoso «como atrás queda tocado». Así pues, tiene otro capítulo dedicado a esto.

El inventor navarro sólo encuentra tres movimientos perpetuos que Dios hizo: el ciclo del agua, el movimiento celeste y el devenir del alma.

"Los ríos caudalosos que continuo corren». Ayanz enuncia correctamente los fundamentos del ciclo hidrológico: "levantan vapores de la tierra, y se condensan en agua, y vuelven a caer en ella, y causa una continua circulación», pero busca su origen en los «planetas e influjos celestes» en lugar de en la energía solar. No comparte la opinión "que otros tienen» de que es igual que la circulación sanguínea. Ve imposible que lo reproduzca el hombre «porque nos falta hacer agentes, como los planetas y la mar, y tierra que haga tal efecto». Ayanz no es el primer español que se preocupa por el ciclo del agua, pues unos años antes, Lastanosa, al que el técnico navarro pudo conocer en la corte ${ }^{7}$, da una explicación similar a este fenómeno en Los veintiún Libros de los Ingenios y de las Máquinas: «No cabe duda (de que el agua) se engendra de exhalaciones (vapores); la cual exhalación llevada encima de los canales de la tierra, y ahí congelada del frío, se convierte en agua, y por causa de su gravedad desciende abajo ${ }^{8}$ ". 
El movimiento celeste, sobre el que afirma que los hombres «ni lo alcanzamos, ni entendemos como se mueven los cielos, ni que materia sean, y cuando la tuviéramos nos faltara lugar». En el Renacimiento, los fundamentos de la astronomía moderna se establecen gracias a las teorías de Copérnico (1473-1543), Tycho Brahe (1546-1601), Kepler (1571-1650) y Galileo (1564-1642) ${ }^{9}$, entre otros, pero probablemente Ayanz no tenga acceso a todas estas fuentes $y$, además, no quiera problemas con la Iglesia, por lo que se limita a pasar de puntillas por tan espinoso asunto.

El «tercero movimiento que será para siempre es el alma, que tenemos hecha a semejanza de Dios». Se trata de una interesante asociación de ideas religiosas y técnicas, propia de una mente analítica preclara.

En cuanto a movimientos perpetuos humanos, Ayanz afirma que ya lo ha tratado previamente en el capítulo sobre "pesar y medir la fuerza de las máquinas», donde hace referencia a la balanza de una barra y una pesa acoplada a una máquina para medir el rendimiento, al que denomina «fatiga» (García Tapia, 2001, pp. 193).

Su idea de las causas del movimiento de las máquinas es muy actual. Influyen lo que llama «peso, número y medida», ahora correspondientes con fuerza, valor y unidad de medida. Para negar la posibilidad de encontrar un móvil perpetuo afirma que no pueden ser continuos los movimientos pues siempre se equilibra el "agente» con el "resistente»; suben, o bajan hasta alcanzar el equilibrio y se necesita una fuerza externa para volver a ponerla en movimiento. Newton, en 1687, enuncia las leyes del movimiento ${ }^{10}$, entre ellas el Principio de acción y reacción que Ayanz parece intuir en el momento en el que cita a un "agente» y a un «resistente».

Ayanz, mediante la prueba, trata de desmontar algunos principios científicos fuertemente asentados. El primero, aquél que afirma «que de una parte de tierra se hacen diez de agua, y de una de agua diez de aire, y de una de aire diez de fuego". Pesando antes la tierra húmeda, secándola y volviéndola a pesar se demuestra que no es así. Así dice: «pesando la tierra, y echándola en el fuego, y después que haya consumido la humedad, tornarla a pesar, y ver lo que menguó». Relaciona este asunto con la generación de minerales por el agua en cuevas, estalactitas y estalagmitas actuales, a las que denomina "torres» y pone el ejemplo de una cueva en Cuenca llamada "de la India». La parte de generación de minerales, ahora cristalización, en minas no puede completarse al faltar una parte en el impreso original.
Para demostrar la parte de «aire» (vapor) que realmente puede generarse del agua, utiliza el aparato dibujado en la figura 3, basado en su máquina de vapor patentada para elevar agua (García Tapia, 1990, pp. 77-90), ahora convertida en un instrumento científico, para conocer cuánta parte de vapor puede salir del agua por medio del calor («fuego»). En este caso, primero ha sido la técnica utilitaria (1606) y luego la experimentación científica (1612).

Se hace un experimento con un cuero que se hincha al recibir el vapor y luego se encoge al condensarse. Tras el ensayo, el autor, Ayanz, parece negar la posibilidad de vacío total, aunque no está claro por faltar parte del impreso original.

\section{«Prosíguese otro modelo sobre la conversión de ayre en fuego»}

"El aire no se convierte en fuego", puesto que "la llama no es fuego sino materia encendida». Es un antecedente de la teoría actual de la combustión, desconocida en la época, en que comúnmente se confundía el fuego con la llama. Se apoya en ejemplos de la naturaleza y hay uno curioso sobre los rayos generados por las nubes en las tormentas que se confundían con fuego y llamas, pero Ayanz dice que no tienen materia. Naturalmente no se conocía su naturaleza eléctrica, pues aún falta más de un siglo para que Franklin ${ }^{11}$ aclare este fenómeno.

Si cambiamos el término "fuerza» por "energía», y "fuego» por "calor», como Ayanz identifica "fuerza» con "fuego" que no tiene materia, resultaría que el "calor» es "energía», descubrimiento revolucionario, si se tiene en cuenta que, aún en el siglo XIX, se hablaba del "calórico ${ }^{12}$ ». También Lastanosa en Los Veintiún libros... trata el tema, aunque de forma más somera: «Mas es al fuego mismo al cual pertenece el calentar en grande manera las $\operatorname{cosas}^{13} »$. A su vez, Gómez Pereira en su obra Antoniana Margarita afirma: «El fuego ínfimo al que denominamos llama [...] se le ha de admitir la acción de calentar» (Barreiro, 2000, pp. 118).

Para Ayanz, la naturaleza de la combustión se demuestra en los volcanes y la lava generada: «el fuego se ha visto salir de algunas bocas de los cóncavos de la tierra, como fue en Sicilia, y en las Indias, que se vio correr por encima de la tierra como un río». Del mismo modo, Gómez Pereira se fija en estos fenómenos geológicos cuando estudia la naturaleza del fuego:

El fuego [...] ocupa su propia región, equiparable a un lugar que no se ve en ningún sitio, salvo en algunas rendijas de la tierra que expulsan llamas (Barreiro, 2000, pp. 118). 
El técnico navarro considera que el fuego puede producir vacío que, al no poder ser total, tiende a ser llenado con otra materia y pone el ejemplo de las ventosas, remedio curativo que producía un vacío que hinchaba la carne sobre la que se aplicaba: "como se ve encendiendo una poquita de estopa en una ventosa, y pegándola a la carne, como no puede haber cosa vacía, todo lo que aquel fuego consume de aire, lo va hinchando la carne». En este caso, se alinea con las tesis del horror vacui ${ }^{14}$, desmontadas por Torricelli (Herrera, 2012, pp. 5) y Pascal (Díez del Corral, 2008, pp. 140).

Ayanz hace un experimento utilizando un vaso de plata o de cobre de muy poco espesor que, dando calor a la caldera a la que se conecta, produce aire (vapor) que al expandirse puede reventar y al condensarse produce vacío y se abolla hacia adentro. No saca conclusiones sobre la presión atmosférica, concepto aún desconocido ${ }^{15}$, pero el experimento va bien encaminado. Desgraciadamente el dibujo (figura 4) está cortado por arriba en el impreso, falta el vaso de plata o de cobre, por lo que el estudio de la experiencia no puede ser completo.

\section{"Lo segundo que ay Esfera de fuego, la qual está} sobre el ayre»

En este apartado el autor trata de desmontar la creencia de la época de la existencia de una «esfera de fuego" por encima de la Luna, basada en buena medida en los pensamientos de Ptolomeo y Aristóteles (Guillaumin, 2005, pp. 151), así como antes Ayanz trató de desmentir la creencia en otras regiones donde se desarrollarían los fenómenos meteorológicos, como el rayo. Por ejemplo, en la segunda parte del Quijote, en la aventura del caballo Clavileño, escrita unos pocos años después por Cervantes (1615), se dice lo siguiente y en boca de Don Quijote:

Sin duda alguna, Sancho, que ya debemos de llegar a la segunda región del aire, adonde se engendra el granizo y las nieves; los relámpagos y los rayos se engendran en la tercera región; y si es que desta manera vamos subiendo, presto daremos en la región del fuego, y no sé yo como templar esta clavija para que no subamos donde nos abrasemos ${ }^{16}$

A pesar del tono irónico de la novela, era cierta la creencia en esta esfera de fuego que seguía representándose en los mapas astronómicos.

Ayanz recurre, como siempre, a la experiencia para desmentir la teoría de la esfera de fuego: pesar y medir cada elemento. Para pesar el aire utiliza una especie de balanza hidrostática y llega a dos conclusiones: "[...] hallé, que las pesas que eran de bronce, metidas en el agua pesan menos que fuera de ella más de dos onzas por libra». Aquí ratifica el principio de Arquímedes y comprueba que el peso de un cuerpo sumergido en un fluido (peso aparente) es inferior.

«[...] hace más fuerza o peso el aire, metido debajo del agua hacia su esfera, o centro, que el agua hacia el suyo». Considera que los elementos tienden a desplazarse a su "centro" y entiende que el peso del aire es mayor que el del agua porque el aire hace más fuerza.

Se pregunta:

El agua por gran viento que haya caerá a tierra, y el aire por más furia que haga una corriente de agua subirá sobre ella, ¿pues porque el fuego ha de ser de menos fuerza, si hubiera esfera donde apeteciera subir?

Concluye que no hay esfera de fuego. Se ve por los cometas que nunca llegan a ella y porque la luz de la esfera de fuego, si existiese, reflejaría en los astros y daría sombras, como el Sol, y no hay tales cosas.

Pero, para poder afirmarlo, recurre de nuevo a una balanza para demostrar que el fuego no pesa, es decir no "apetece» a su centro porque no lo tiene, ni tiene materia. Es solo «calor», es decir «energía», empleando el término actual.

\section{«Prosíguese sobre lo mismo de la esfera de fuego»}

Ayanz insiste en el tema siguiendo su idea, comenzando con una contundente frase que revela su admirable mentalidad científica, surgida de su experiencia como inventor: "Ninguna filosofía hay más cierta que la prueba, porque ella es la que nos satisface y convence a nuestras opiniones». Con esta sentencia, Ayanz suscribe las palabras de Leonardo, quien afirma:

No saben que mis materiales tienen más valor porque derivan de la experiencia antes que de las palabras de otros, y la experiencia es la maestra de quienes han escrito con acierto ${ }^{17}$

Estos pensamientos, junto a otros similares, van sentando las bases de las corrientes empíricas surgidas en la Edad Moderna.

Siguiendo este principio, recurre a dos de sus instrumentos científicos favoritos: la balanza y la caja cerrada (figura 7) a donde se hace llegar el fuego y el aire (vapor). De esta forma confirma, una vez más, la falta de peso del fuego y vuelve a aseverar que «no hay esfera de fuego». 
Las diferentes temperaturas entre zonas y entre el invierno y la época estival eran explicadas por la presencia de una esfera de fuego. En relación a esto, Gómez Pereira explica que:

[El fuego] manifiesta su potencia y dominio más extenso y más próximo a nosotros, en las épocas de calor estival por la influencia del sol y de otras estrellas que favorecen su calor y sequedad [...]; en la época invernal se retira a un lugar estrecho por el influjo de otras estrellas contrarias que favorecen la frialdad (Barreiro, 2000, pp. 117).

Ayanz atribuye las diferencias térmicas a la variación en la inclinación de los rayos del Sol, lo cual, sabemos ahora, es cierto. Así, el técnico navarro concluye:

Y se ve claro, que esta destemplanza de calor, o frío lo hace el Sol, el estar más desviado, y dilatarse más el día o la noche.

Tras negar la existencia de la esfera ígnea, al técnico navarro le sobreviene otra duda, ¿de dónde viene el fuego? Ayanz lo sitúa en el centro de la tierra, de donde vienen los volcanes. Explica que para crearlo se necesita una materia combustible, porque el fuego no es materia, ni tiene peso, ni ocupa lugar, es sólo "fuerza» (energía). Es más, completando su teoría sobre las piedras imantadas ${ }^{18}$ deduce que el fuego es de la misma naturaleza que el imán que extiende su «virtud» (magnetismo) a otras piedras imantadas. Ahora diríamos que el "calor» (fuego) y el "magnetismo» son formas de la "energía», que se transmite de unas materias a otras. Aquí Ayanz demuestra su capacidad de precursor una vez más, aunque comete errores, como cuando afirma que el Sol es solo «fuego», sin cuerpo ni peso.

Finalmente, concluye con otra muestra de su racionalidad científica:

Los que dicen que está [la esfera de fuego] se fundan en

lo que oyeran decir, sin ninguna prueba ni demostración.

\section{«La tercera opinión, que los elementos compelen unos a otros»}

El término "compeler» equivale a «desplazar» y aquí desmiente otra opinión de la época, según la cual los elementos obligaban por sí mismos a desplazarse unos a otros. Para Ayanz esto es falso y es una fuerza exterior la que los desplaza. En la naturaleza pone el ejemplo de los terremotos en los que la tierra se desplaza por una fuerza, la del aire a presión en las oquedades de la Tierra. Así, dice: «el aire encerrado en los cóncavos de la tierra, que va creciendo, y por no caber donde está, la hace temblar, hasta que la revienta y sale».
Para Aristóteles, la velocidad de caída de los cuerpos depende de su peso y de la resistencia del medio (Romo, 1985, pp. 25), motivo por el cual los cuerpos pesados caen más rápido que los ligeros (Mason, 2012, pp. 230). Esta idea fue aceptada hasta que Simon Stevin (1548-1620) demuestra que no era así. En un experimento atribuido erróneamente a Galileo (Mason, 2012, pp. 230) explica:

Tomemos [...] dos balas de plomo, una de ellas diez veces mayor de peso que la otra, que dejaremos caer juntas de la altura de treinta pies [...], y se verá que la más ligera no emplea diez veces más tiempo para caer que la más pesada, sino que caen con tanta igualdad sobre la plancha que ambos ruidos parecen una única sensación de sonido ${ }^{19}$.

Previamente, en España, Lastanosa propone sus teorías de la caída de los graves, siendo estas similares a las de Stevin. Así dice: «Cuanto de más alto bajan los pesos, tanta más velocidad y presteza traen consigo ${ }^{20} \|$, y añade:

[De] dos pesos que descienden [que] sean de la misma materia y de una misma forma y de un mismo peso, hace mayor golpe el que cae de más alto ${ }^{21}$.

Ayanz da también su explicación de la caída de los graves:

[La razón de ello] no procede sino de su mismo peso que, como apetece su centro, que cuanto más alto cae, va tomando más fuerza hasta que cae a su centro.

Para el inventor navarro tampoco es el aire el que impulsa al cuerpo que cae, sino al contrario, hace resistencia. Ilustra su explicación con el ejemplo de un águila que abre las alas y el aire frena su caída.

Para demostrarlo, de nuevo a través de la experimentación, mide con una balanza al aire libre el peso de un cuerpo que cae y luego lo hace con el mismo cuerpo en un cántaro donde el aire está confinado, comprobando que en el primer caso hay mayor resistencia del aire y, por tanto, el peso es menor. Esta opinión la sustancia con varios ejemplos para demostrar que nunca hay «compulsión de elementos».

Finalmente utiliza esto para unir la negación del movimiento perpetuo, con la de la compulsión de elementos. Para ello fabrica una bomba de rosario de cajas (figura 9) que van dentro de un cilindro. Si hubiese compulsión de elementos (agua y aire) funcionaría como una máquina de movimiento perpetuo, pero, en la práctica, hace falta un agente exterior (una persona que mueva la manivela de la bomba). Aunque es cierto que se trata de una bomba de mayor rendimiento, nunca podrá ser de movimiento continuo. 


\section{CONCLUSIONES}

Ayanz escribe esto en una época (principios del siglo XVII) y en un ambiente en que España se aleja de la revolución científica que se iniciaría en Italia con Galileo. Pero este capítulo, junto con las invenciones españolas, demuestra que en España había círculos técnicos y científicos que trataban de desarrollar estas disciplinas. Ciertamente, se trataba de casos aislados, fuera de las universidades y de los círculos de poder políticos y eclesiásticos. El ejemplo de las notas al margen del impreso, escritas posiblemente por un jesuita, demuestra el rechazo que producía todo lo que no siguiese una línea de pensamiento rígidamente establecida.

En este caso Ayanz desmonta una serie de tópicos como el movimiento perpetuo, la naturaleza del fuego como elemento, la caída de los graves, la existencia de la esfera de fuego o la compulsión de elementos. Además lo hace sólidamente, apoyando sus afirmaciones o, más bien, sus negaciones, no solo en observaciones de la naturaleza, sino en experiencias de laboratorio, con instrumentos basados en máquinas que él había inventado y patentado. En este aspecto, los resultados son verdaderamente innovadores.

Aunque Ayanz trata de sortear los tópicos más sensibles a la prohibición eclesiástica, no por ello deja de desmontar creencias que podrían molestar, como así parece que fue, a los poderes religiosos de la época ¿Fue por eso el libro prohibido, o simplemente no tuvo tiempo de imprimirlo completo al Ilegarle la enfermedad y la muerte?

Por desgracia, al menos en este capítulo que conocemos, no llegan a formularse teorías claras que sustituyan a las creencias desmontadas. Se desmiente la existencia de la esfera de fuego, pero no se llega a formular un sistema planetario, se limita a decir que "no lo entendemos», quizá por el riesgo de atentar a la autoridad religiosa que, Ayanz, hombre creyente, respetaba. Se desmiente la naturaleza material del fuego como elemento, pero, aunque se inicia, no se establece de forma clara una teoría de la combustión. Se desmonta la idea de la "compulsión» de elementos como explicación de la caída de un grave, pero no se formula una ley clara de la gravedad. Finalmente, aunque hay más cosas, se demuestra rotundamente la imposibilidad del movimiento perpetuo, pero se admiten algunos "creados por Dios", y no se explican con claridad, aunque se intuyen las causas del rozamiento que lo impiden. Ciertamente, todo ello es un gran avance respecto a la ciencia de la época, pero lejos aún de las formulaciones de su contemporáneo Galileo.

El fuerte de Ayanz sigue siendo la tecnología, profusamente demostrado en sus asombrosas invenciones. Esta experiencia técnica le lleva a la demostración de sus afirmaciones a través de los instrumentos técnicos que él había fabricado. Por ejemplo, se intuye que el fuego es energía, como había comprobado en su máquina de vapor. Falta, en cambio, el cálculo matemático y se echa de menos la fórmula, aunque sea simple, que cuantifique los resultados de su experiencia. Sin embargo, Ayanz había tenido en su juventud, como paje del rey, una formación en la Escuela de Matemáticas para pajes ¿Estarán en otros capítulos que desconocemos?

Como vemos, estas conclusiones abren varios interrogantes que dejamos para otros investigadores. Lo que hemos pretendido es presentar una nueva faceta que Ayanz desarrolló en los últimos años de su vida: la de científico que completa la compleja personalidad de un personaje que cultivó la milicia, la política, la gestión empresarial y administrativa, las artes, la técnica, la invención y cuya carrera culmina con la investigación experimental científica. 
Figura 1. Hoja 1 del impreso. Fuente: Biblioteca Nacional, Al Serenissimo Príncipe... R/34183/10.

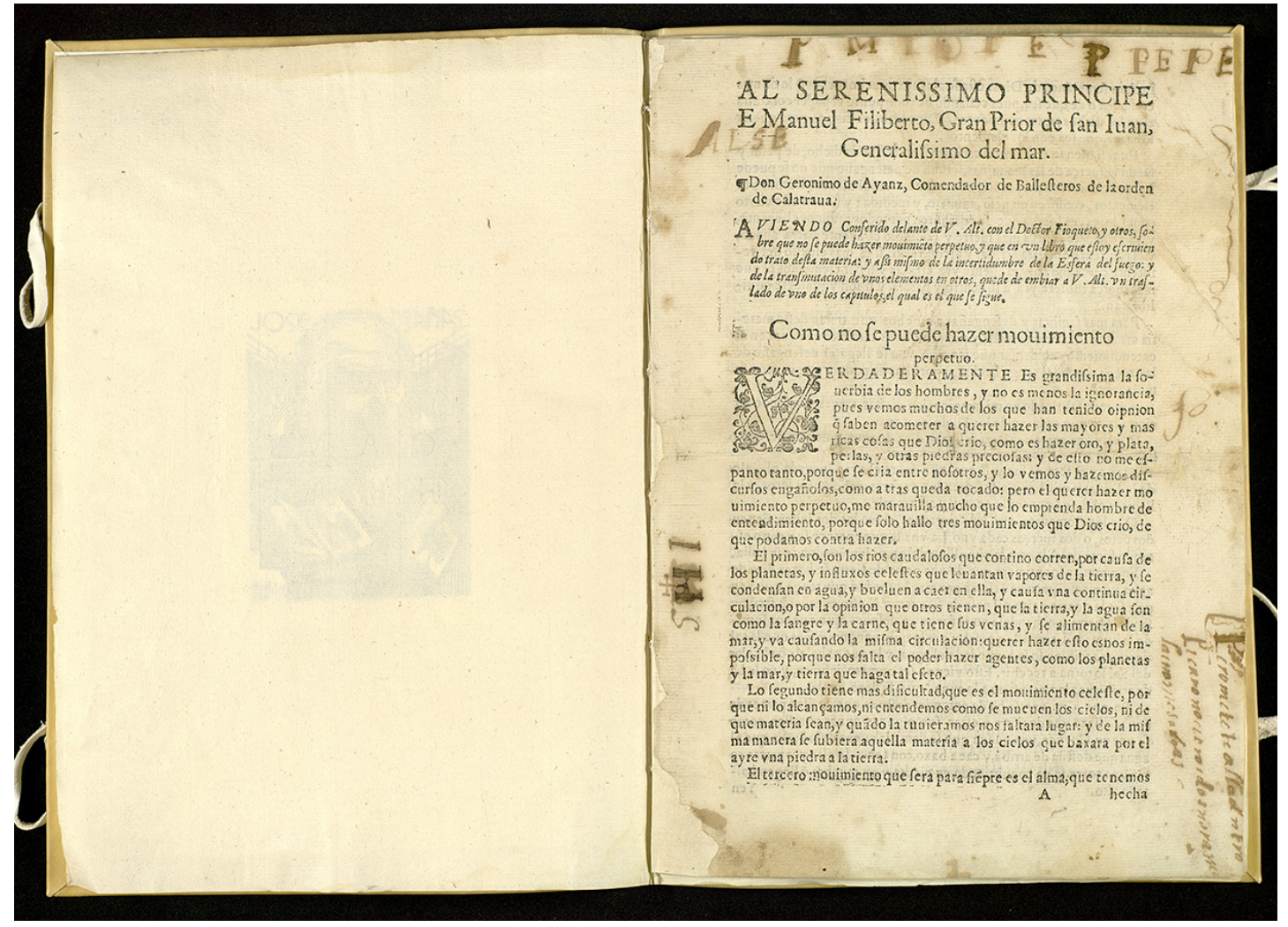

Figura 2. Hoja 2 del impreso. Fuente: Biblioteca Nacional, Al Serenissimo Príncipe... R/34183/10.

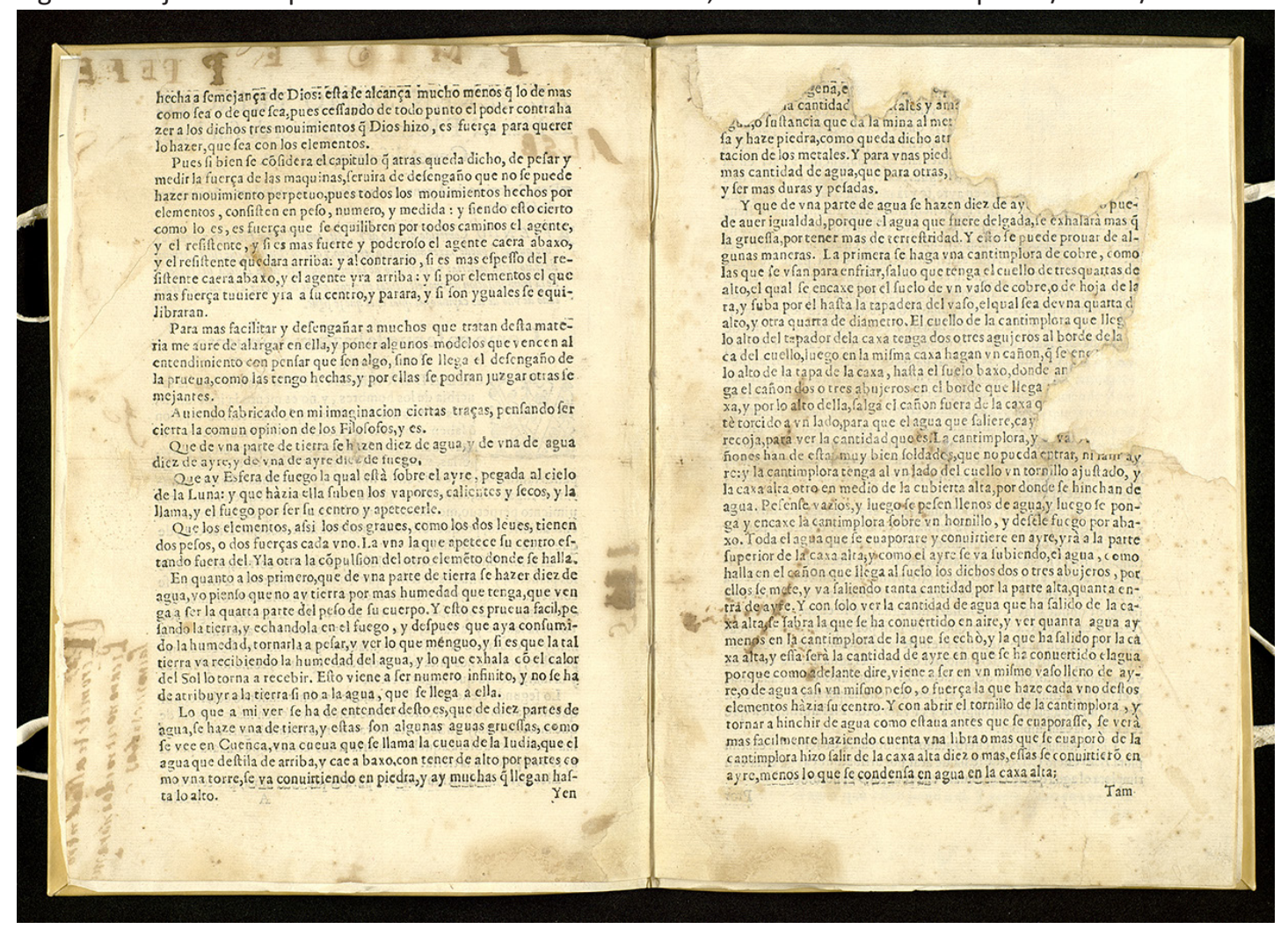


Figura 3. Hoja 3 del impreso. Fuente: Biblioteca Nacional, Al Serenissimo Príncipe... R/34183/10.

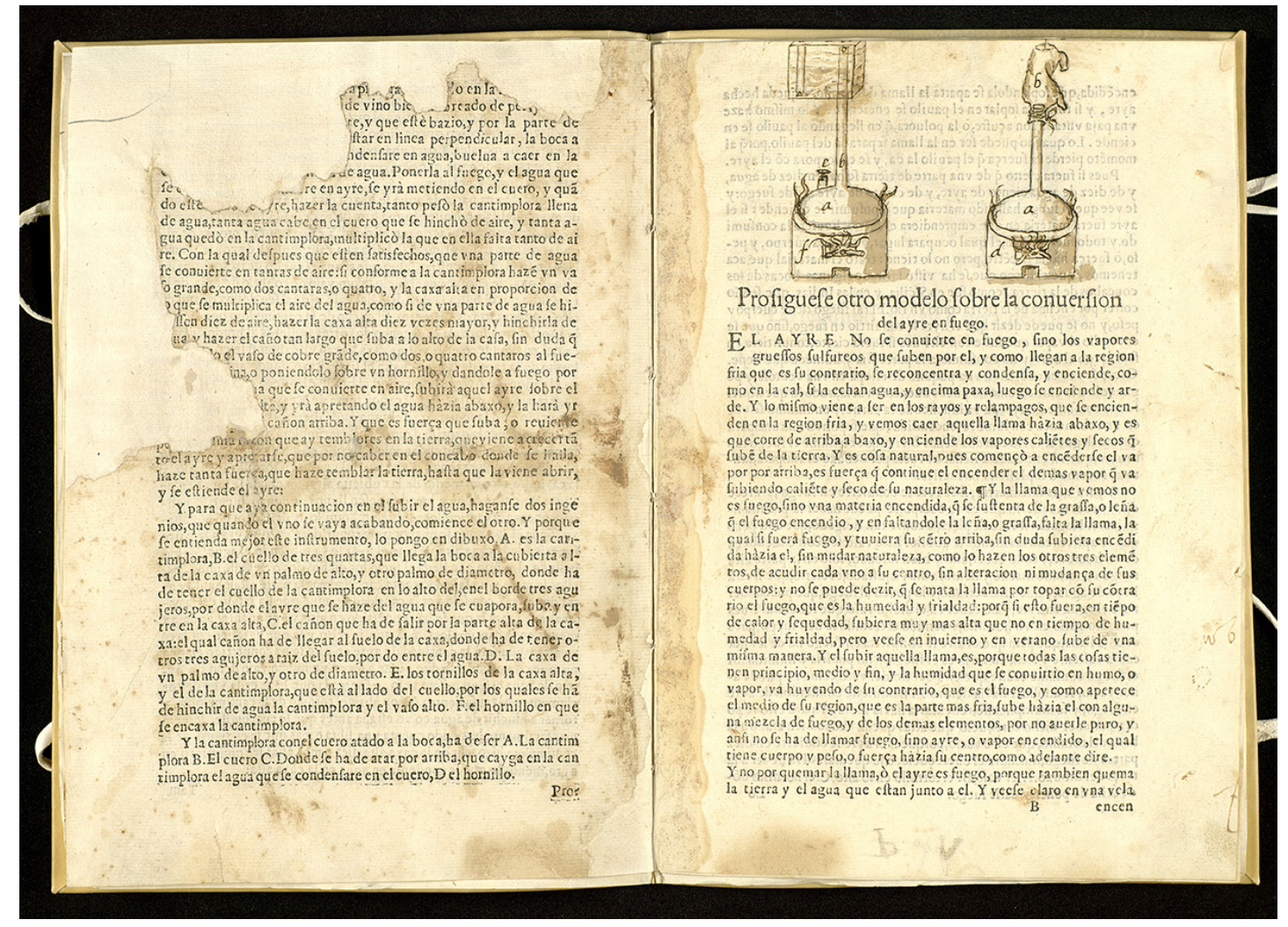

Figura 4. Hoja 4 del impreso. Fuente: Biblioteca Nacional, Al Serenissimo Príncipe... R/34183/10.

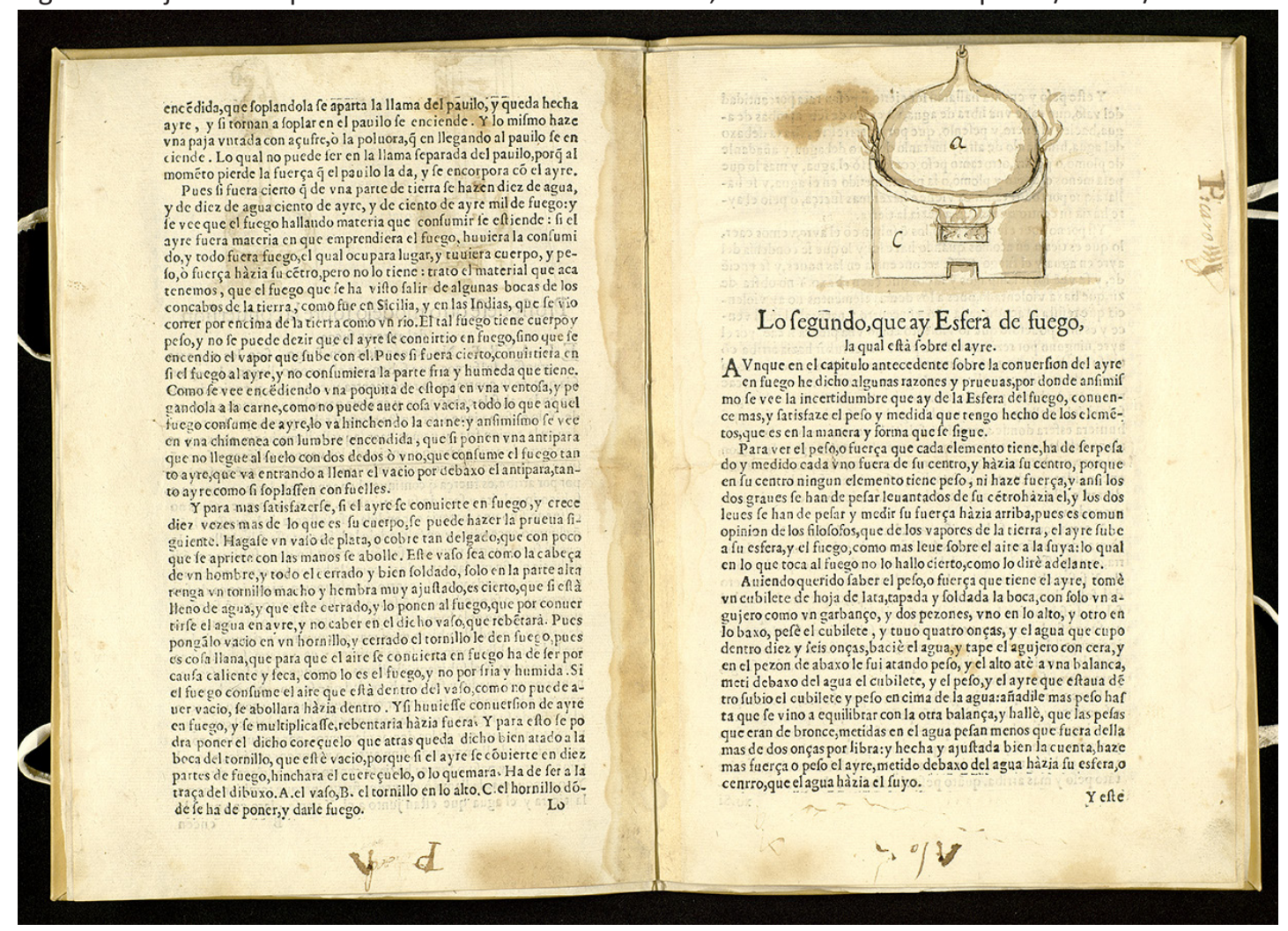


Figura 5. Hoja 5 del impreso. Fuente: Biblioteca Nacional, Al Serenissimo Príncipe... R/34183/10.

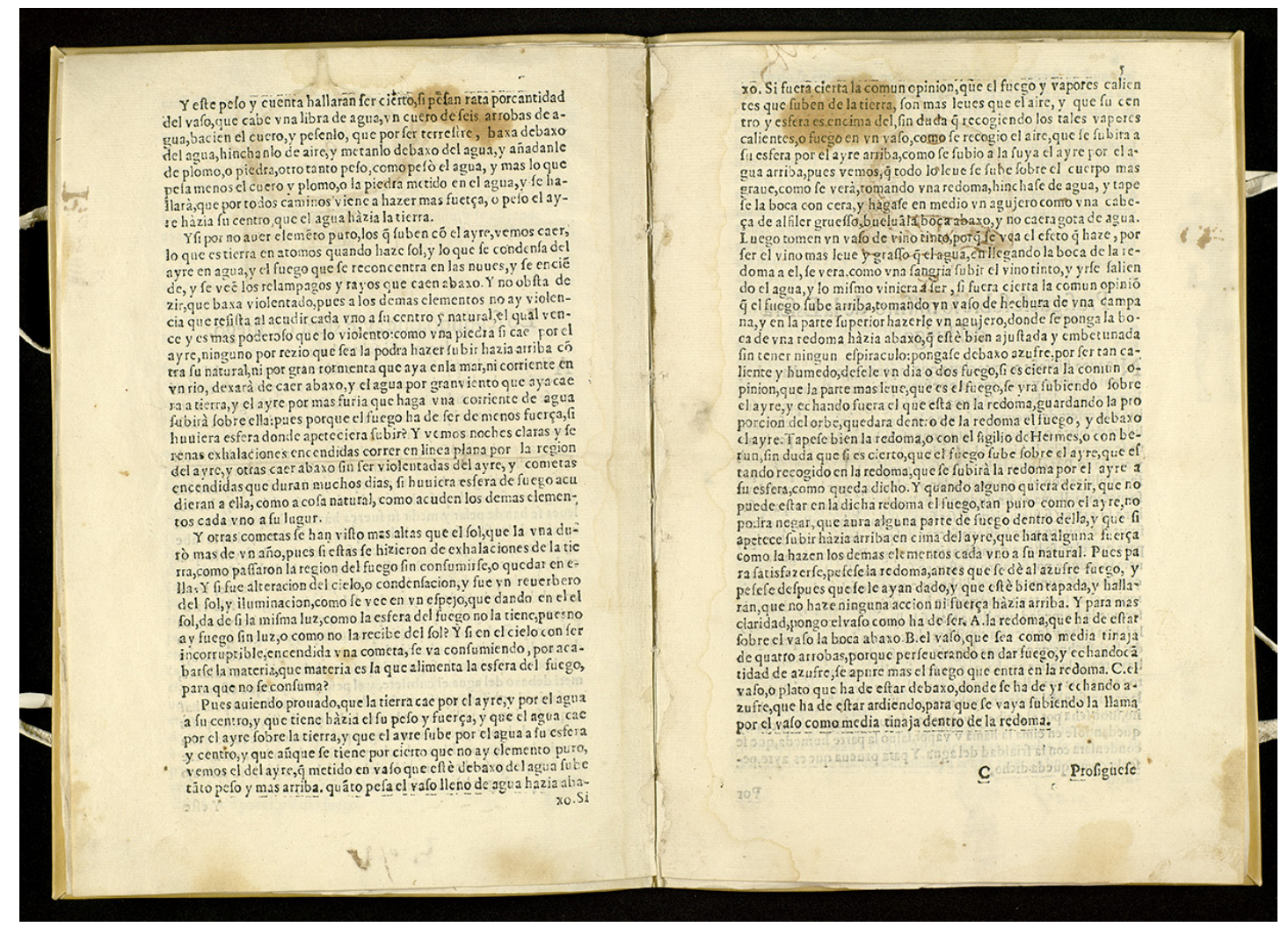

Figura 6. Hoja 6 del impreso. Fuente: Biblioteca Nacional, Al Serenissimo Príncipe... R/34183/10.

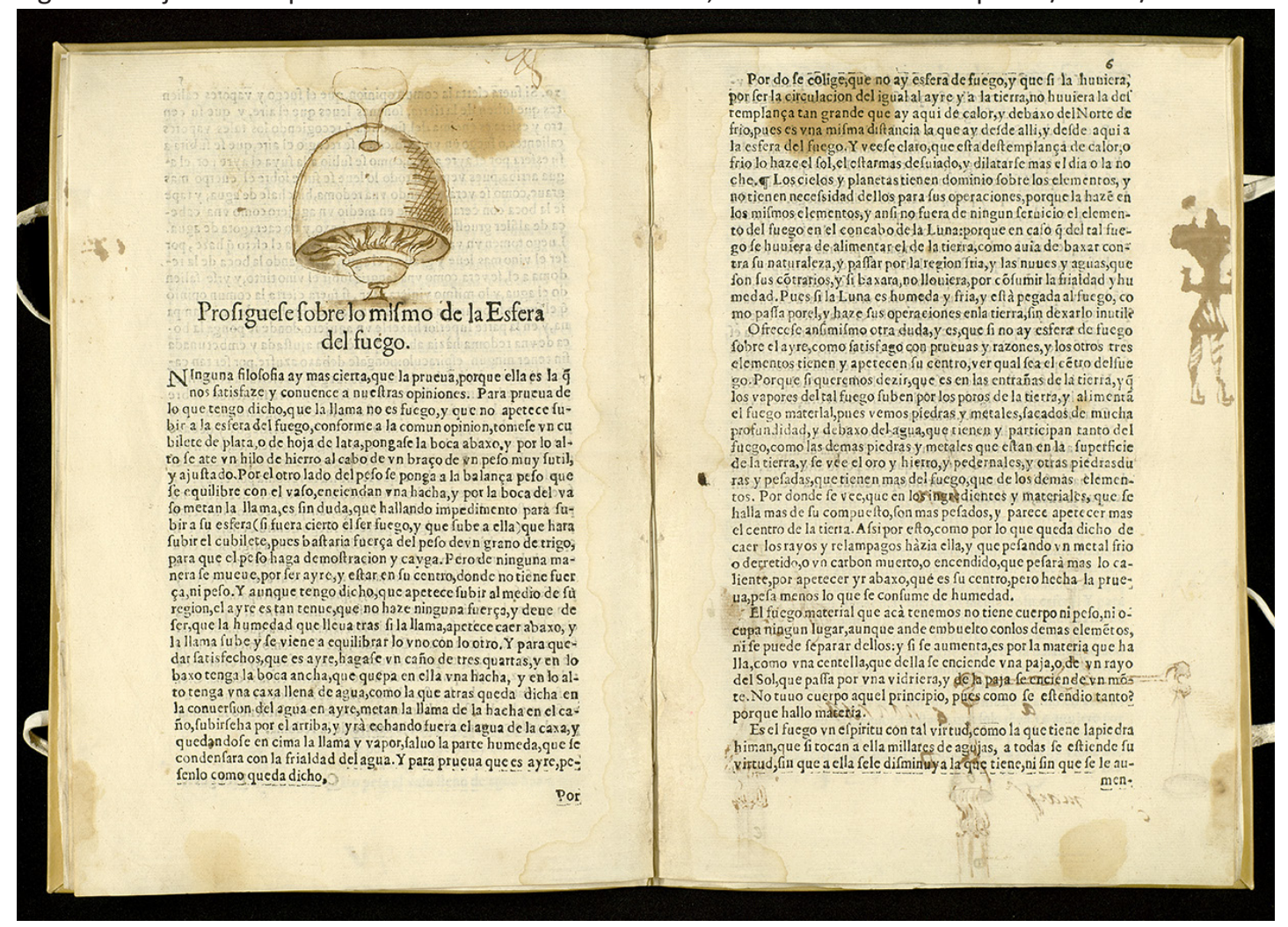


Figura 7. Hoja 7 del impreso. Fuente: Biblioteca Nacional, Al Serenissimo Príncipe... R/34183/10.

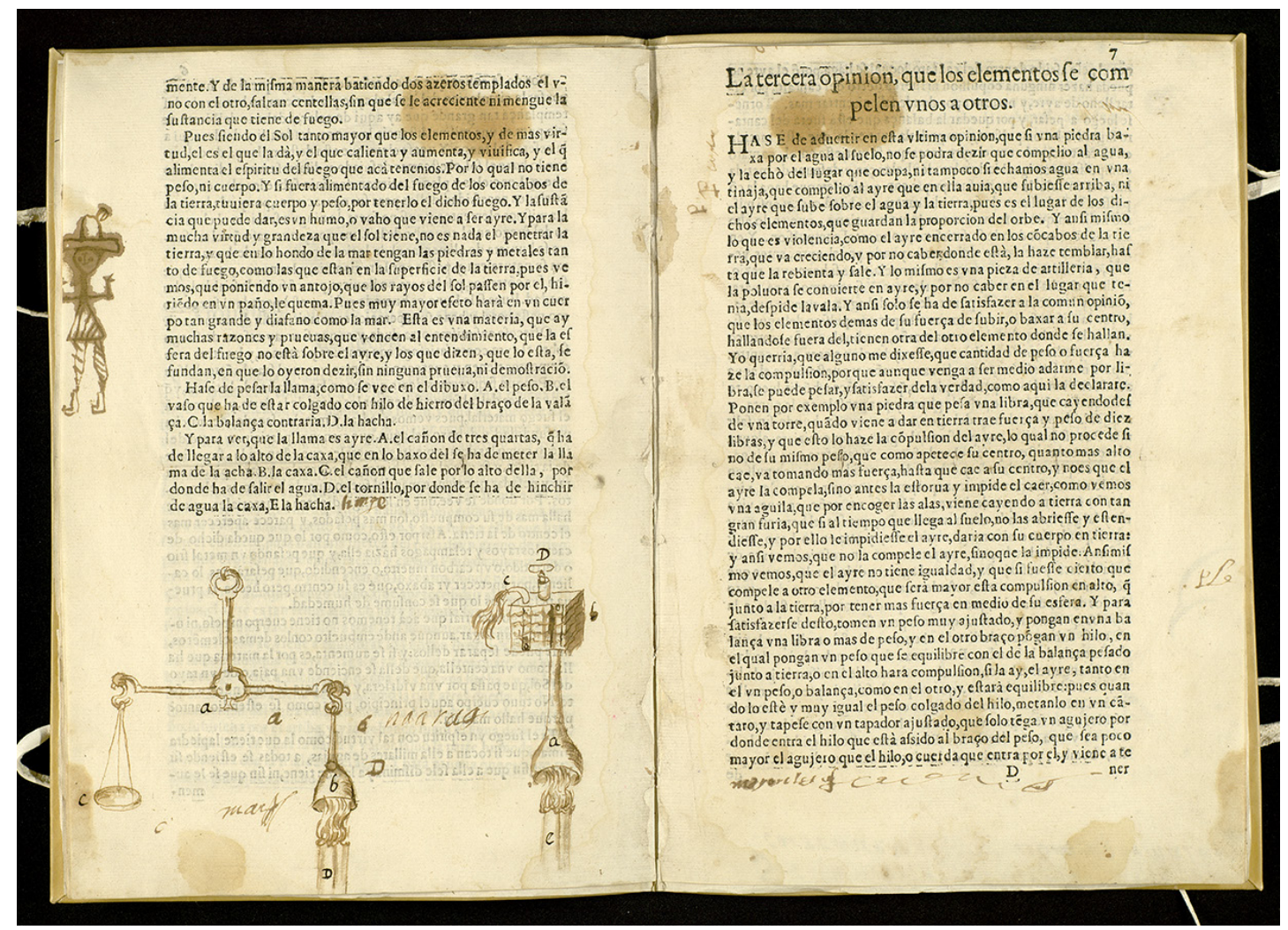

Figura 8. Hoja 8 del impreso. Fuente: Biblioteca Nacional, Al Serenissimo Príncipe... R/34183/10.

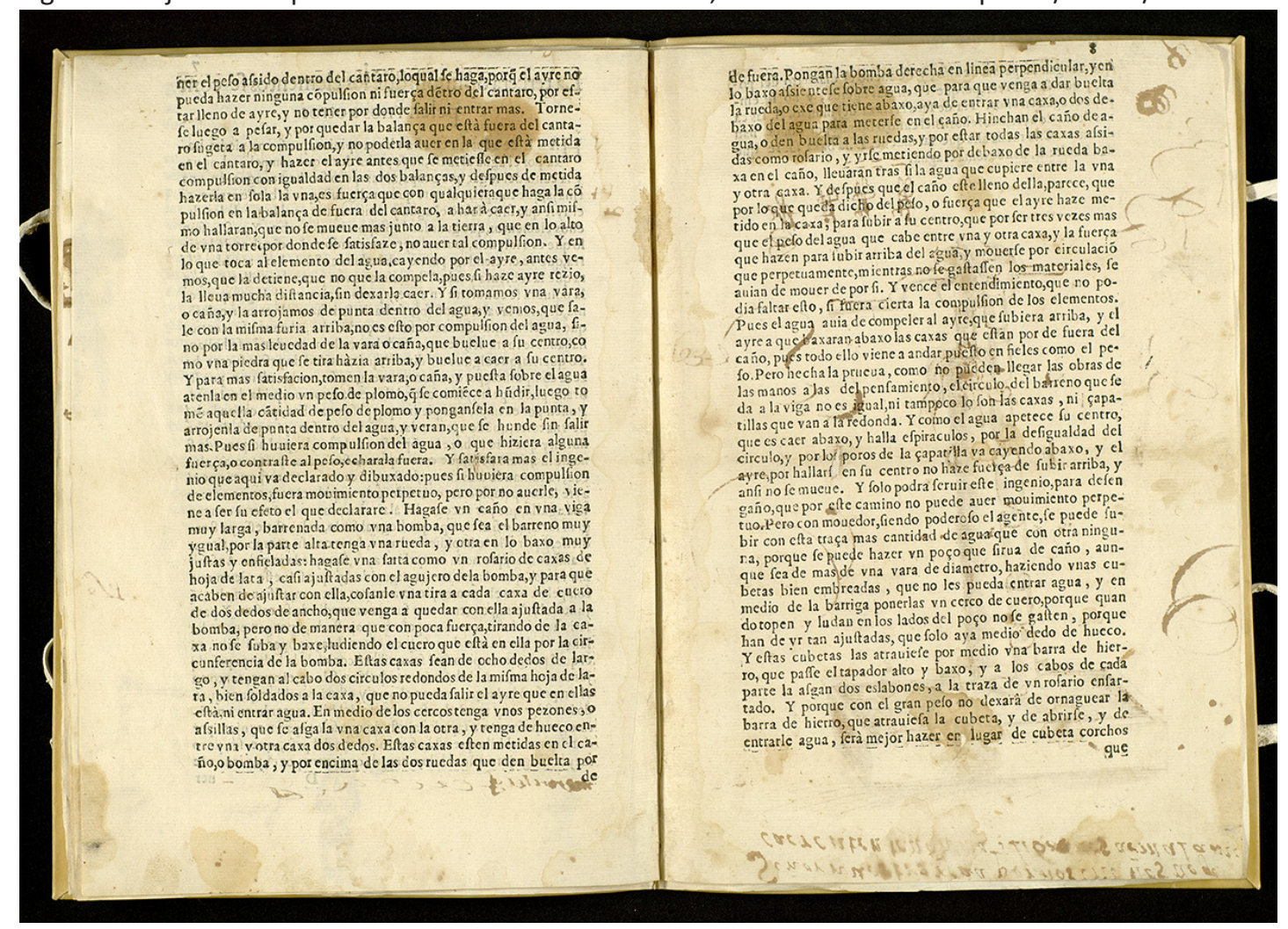


Figura 9. Hoja 9 del impreso. Fuente: Biblioteca Nacional, Al Serenissimo Príncipe... R/34183/10.

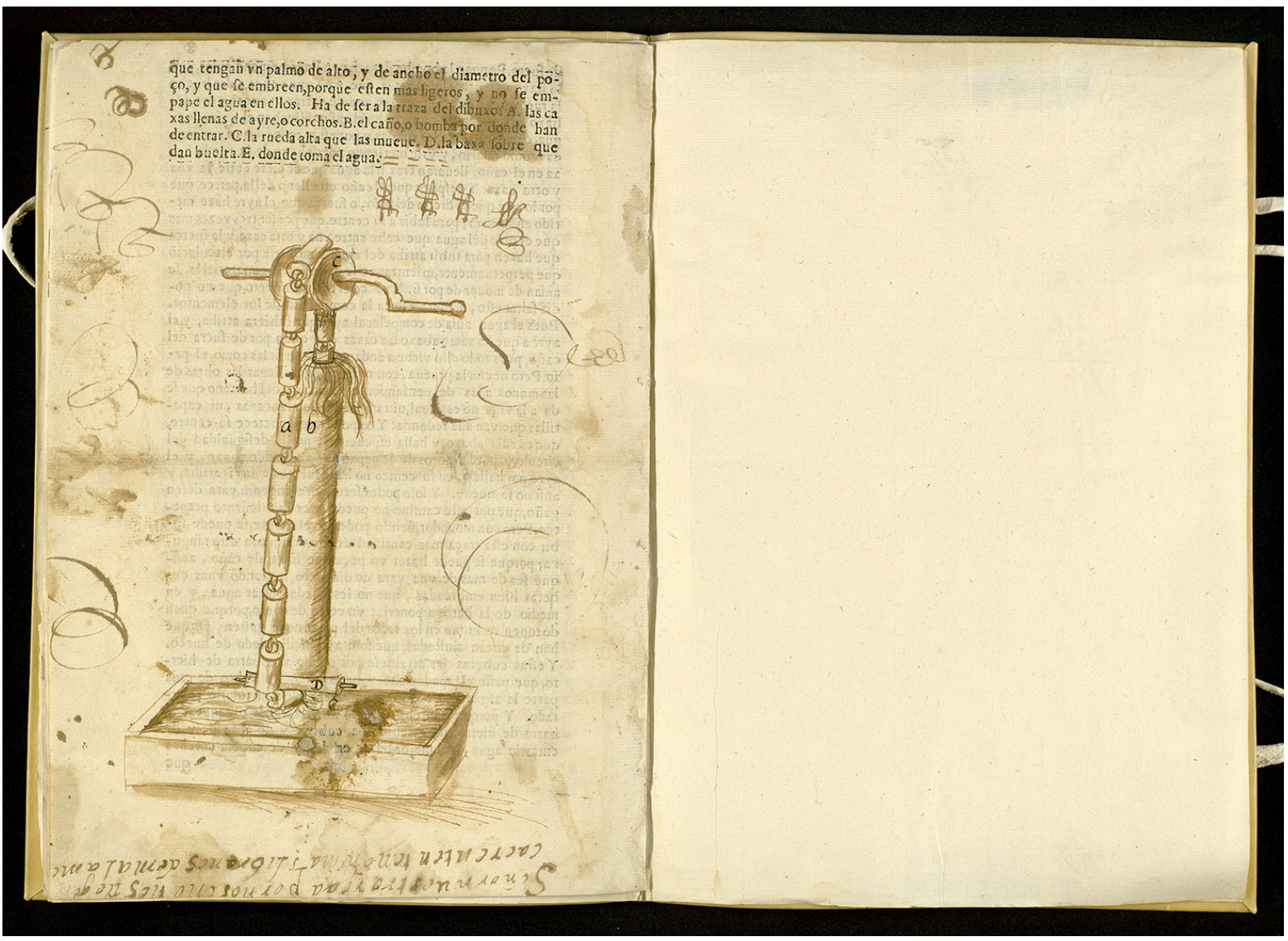

\section{NOTAS}

1 Ayanz y Beaumont, Jerónimo (1612), Al Serenissimo Principe Emanuel Filiberto, Gran Prior de San Juan, Generalissimo del mar. Don Gerónimo de Ayanz... sobre que no se puede hazer movimie[n] to perpetuo... y assi mismo de la incertidumbre de la Esfera del fuego y de la transmutación de unos elementos en otros. Biblioteca Nacional de España, Madrid, R/34183/10.

2 En el Archivo de Simancas, sección Cámara de Castilla, con el número de inventario 174, se encuentra un Libro de Cédulas de 440 folios, encuadernado en pergamino, con los más diversos asuntos. Dentro de este libro se encuentra una Cédula Real, firmada por el rey Felipe III con el título "Gerónimo de Ayanz", otorgando a este demandante un privilegio para disfrutar del derecho exclusivo de unas cincuenta invenciones, cuyos dibujos y explicaciones revisten un alto interés. La citada Cédula de Privilegio ocupa los folios 49v. al 94, y está fechada el 1 de septiembre de 1606, en San Lorenzo de El Escorial.

3 Fernández Navarrete, Martín, Colección de documentos, tomo XXII, fols. 142-145.

4 Jerónimo de Ayanz, Respuesta..., Archivo General de Simancas, Contadurías Generales, leg. 854, fols. 626-636.
5 Título de Capitán General de la Mar al Serenísimo Príncipe Emanuel Filiberto, Gran prior de San Juan. (Enero de 1612). Fernández Navarrete, Martín, Colección de documentos, tomo III, fol. 57.

6 Con toda seguridad se trata de Gian Francesco Fiochetto (1564-1642), protomédico de Carlo Emanuele I de Saboya (1562-1630), padre del destinatario de la carta, Emanuel Filiberto de Saboya. Véase: Trompeo, Benedetto (1867). Notizie storiche e biografiche intorno al conte Gian Francesco Fiochetto, protomedico generale, archiatro de S.A.S. II Duca Carlo Emanuele Primo di Savoia. Torino. Tipografia Nazionale di Bottero Luigi.

7 En el periodo en que Ayanz fue paje real (1567-1571) (García Tapia, 2001, pp. 45-47) pudo entrar en contacto con dos grandes técnicos, Lastanosa y Turriano. El primero es el revisor de los ingenios que se presentan al rey solicitando el privilegio de invención (García Tapia y Carrillo Castillo, 2002, pp. 63) y, a la vez, está trabajando en la redacción del tratado más importante de ingeniería hidráulica hasta el Renacimiento, Los Veintiún Libros de los Ingenios y de las Máquinas (García Tapia, 1997, pp. 180). Por su parte, Turriano, con el que Ayanz mantiene una 
buena relación (García Tapia, 2001, pp. 46), construye en este periodo su célebre artificio en Toledo (García Tapia y Carrillo Castillo, 2002, pp. 40). Es de esperar que la personalidad y las ideas de estos técnicos calasen en el joven Ayanz.

8 Lastanosa, Pedro Juan de (c. 1570), Los Veintiún Libros de los Ingenios y de las Máquinas de Juanelo. Libro 1, fol. 8r.

9 Véanse los capítulos "El sistema del mundo copernicano" y "Galileo y la ciencia de la mecánica" en Mason, Stephen (2012), Historia de las ciencias 1: Desde la antigüedad hasta el siglo XVII, Madrid, Alianza, pp. 191-207 y pp. 223-248.

10 Las célebres leyes del movimiento son publicadas en 1687 en la obra Philosophiae naturalis principia mathematica de Isaac Newton.

11 Benjamin Franklin publica en The Pennsylvania Gazette en octubre de 1752 un experimento con el que demuestra la naturaleza eléctrica del rayo.

12 El "calórico" es un fluido hipotético que impregna a los cuerpos y pasa desde sustancias calientes a sustancias frías. Véase: Lavoisier, Antoine Laurent (1777), "Réflexions sur le phlogistique, pour servir de développement à la théorie de la combustion \& de la calcination, publiée en 1777". Mémoires de l'Académie des Sciences, année 1783. El mérito de Ayanz en este aspecto radica en que no se pudo negar la existencia del calórico hasta los experimentos de B. Thomson y H. Davy a finales del siglo XVIII, véase: García Colín, Leopoldo (2008), De la máquina de vapor al cero absoluto: calor y entropía, México D.F., Fondo de cultura antigua, 21.

\section{BIBLIOGRAFÍA}

Barreiro Barreiro, José Luis (2000), Reproducción facsimilar de la edición de 1749 de Antoniana Margarita de Gómez Pereira. Santiago de Compostela y Oviedo, Universidad de Santiago de Compostela y Fundación Gustavo Bueno.

Díez del Corral, Francisco (2008), Blaise Pascal: la certeza y la duda. Madrid, Vision Net.

Fernández Duro, Cesáreo (1885), El Gran Duque de Osuna y su marina. Reimpreso en Madrid (2006), Renacimiento.

García-Diego, José Antonio; García Tapia, Nicolás (1990), Vida y técnica en el Renacimiento. Manuscrito de Francisco Lobato, vecino de Medina del Campo. Valladolid, Universidad de Valladolid.

García Tapia, Nicolás (1990), Patentes de invención españolas en el Siglo de Oro. Madrid, Ministerio de Industria y Energía.

García Tapia, Nicolás (1992), Del dios del fuego a la máquina de vapor: introducción de la técnica en Hispanoamérica. Valladolid, Ámbito Ediciones S.A.

García Tapia, Nicolás (1997), Los Veintiún libros de los ingenios y de las máquinas, atribuidos a Pedro Juan de Lastanosa. Zaragoza, Gobierno de Aragón.
13 Lastanosa, Pedro Juan de (c. 1570), Los Veintiún Libros de los Ingenios y de las Máquinas de Juanelo. Libro 1, fol. 14r.

14 La teoría del horror vacui está basada en las ideas de Aristóteles que en su tratado "Física" hace una amplia disertación sobre la imposibilidad de la existencia del vacío.

15 El primer ensayo conocido que demuestra la existencia de la presión atmosférica lo realiza el físico italiano Evangelista Torricelli. Este experimento no fue publicado como tal, pero sabemos de su existencia por el contenido de una carta dirigida a Michelangelo Ricci, fechada el 11 de junio de 1644, en la que explica la experiencia y las conclusiones de la misma.

16 Fragmento de El Quijote, parte II, capítulo XLI.

17 Leonardo da Vinci, Códice Atlántico, folio 327v.

18 Véase el capítulo Ayanz y la llamada "Aguja fija" de Luis da Fonseca en: García Tapia, Nicolás (2001), Un inventor navarro, Jerónimo de Ayanz y Beaumont, Pamplona, Gobierno de Navarra, pp. 237-240

19 En 1586, Stevin publica una obra de Mecánica que contenía conclusiones importantes, entre otras, la del experimento sobre la velocidad de caída de los cuerpos.

20 Lastanosa, Pedro Juan de (c. 1570), Los Veintiún Libros de los Ingenios y de las Máquinas de Juanelo. Libro 18, fol. 386r.

21 Lastanosa, Pedro Juan de (c. 1570), Los Veintiún Libros de los Ingenios y de las Máquinas de Juanelo. Libro 18, fol. 386r.

García Tapia, Nicolás (2001), Un inventor navarro, Jerónimo de Ayanz y Beaumont. Pamplona, Gobierno de Navarra.

García Tapia, Nicolás; Carrillo Castillo, Jesús (2002), Turriano, Lastanosa, Herrera, Ayanz. Tecnología e imperio. Ingenios y leyendas del Siglo de Oro. Madrid, Nivola.

Guillaumin, Godfrey (2005), El surgimiento de la noción de evidencia: un estudio de epistemología histórica sobre la idea de evidencia científica. México D.F., Universidad Nacional Autónoma de México.

Herrera, Rosa María (2012), “Historia del experimento barométrico", Pensamiento matemático, 2, pp. 1-14, 5.

Mason, Stephen (2012), Historia de las ciencias 1: Desde la Antigüedad hasta el siglo XVII. Madrid, Alianza.

Navarro Brotóns, Víctor (1996), “Los Jesuitas y la renovación científica en la España del siglo XVII", Studia historica. Historia moderna, 14 (1), pp. 15-44, 16.

Romo Feito, José (1985), La física de Galileo: la problemática en torno a la ley de caída de los cuerpos. Barcelona, Universidad Autónoma. 